\title{
Traitement d'imprévus et « modèles de professionnalité en actes » lors d'une séance de grammaire au cycle 3 : étude de cas d'une enseignante stagiaire à l'école primaire
}

Treatment of didactic unforeseen events and "models of professionality in acts" during a "cycle 3" grammary lesson : case study of a trainee teacher at Primary school

\section{Nicole Bénaïoun-Ramirez}

\section{OpenEdition}

\section{Journals}

Édition électronique

URL : https://journals.openedition.org/educationdidactique/2086

DOI : 10.4000/educationdidactique.2086

ISSN : $2111-4838$

Éditeur

Presses universitaires de Rennes

Édition imprimée

Date de publication : 20 décembre 2014

Pagination : 95-109

ISBN : 978-2-7535-4002-6

ISSN : 1956-3485

Référence électronique

Nicole Bénaïoun-Ramirez, «Traitement d'imprévus et « modèles de professionnalité en actes » lors d'une séance de grammaire au cycle 3 : étude de cas d'une enseignante stagiaire à l'école primaire », Éducation et didactique [En ligne], 8-3 I décembre 2014, mis en ligne le 20 décembre 2016, consulté le 23 août 2022. URL : http://journals.openedition.org/educationdidactique/2086 ; DOI : https://doi.org/ 10.4000/educationdidactique.2086 


\title{
TRAITEMENT D'IMPRÉVUS ET « MODÈLES DE PROFESSIONNALITÉ EN ACTES * LORS D'UNE SÉANCE DE GRAMMAIRE AU CYCLE 3 : ÉTUDE DE CAS D'UNE ENSEIGNANTE STAGIAIRE À L'ÉCOLE PRIMAIRE
}

Nicole Bénaïoun-Ramirez

UMR EFTS, université de Toulouse Jean Jaurès

\begin{abstract}
La formation professionnelle des professeurs des écoles connaissant de profondes transformations, il est essentiel d'analyser les pratiques et les représentations de professeurs débutants.

Cet article est une étude de cas (entretiens et pratiques constatées) qui se propose d'analyser les pratiques d'enseignement de la langue (premier degré) d'une enseignante débutante face aux imprévus didactiques dont le traitement renseigne sur ses modèles de référence. Quelles représentations professionnelles de l'enseignement peuvent être inférées à partir des entretiens et du traitement d'imprévus didactiques rencontrés pendant sa séance ? En quoi des « modèles de professionnalité en actes » pourraient-ils mettre en tension ces représentations?
\end{abstract}

Mots-clés : Imprévu didactique, incident cognitif, modèles de professionnalité en actes, représentations professionnelles, formation professionnelle initiale.

Treatment of didactic unforeseen events and « models of professionality in acts" during a "cycle 3" grammary lesson: case study of a trainee teacher at Primary school

When the vocational training of Primary school teachers knows profound transformations, we have to analyze the practices and the representations of novice professors.

The article is a case study (interviews and noted practices) which intends analyzing the first degree language teaching practices of a novice teacher who has to face didactic unforeseen events. Their treatment informs about its benchmark models. Which professional representations of the teaching can be deduced from the interviews and from the treatment of didactic unforeseen events met during her lesson? In what ways could "models of professionnality in acts " be at variance with these representations?

Keywords: Didactic unforeseen event, cognitive incident, models of professionality in acts, professional representations, initial vocational training. 
Nicole Bénaïoun-Ramirez

La formation professionnelle des professeurs des écoles connaissant de profondes transformations, il est essentiel d'analyser les pratiques et les représentations de professeurs débutants. Nous avons recueilli des données en 2008, au moment où la mastérisation allait se mettre en place, auprès de Professeurs des Écoles Stagiaires (PES) qui avaient, comme les PES actuels, des périodes de Pratique Accompagnée (PA) dans les classes des Professeurs des Écoles MaîtresFormateurs (PEMF).

Les PES qui ont réussi le concours de recrutement des professeurs des écoles, ceux de 2008 comme les PES actuels, ne sont plus des étudiants mais des " professeurs en formation » qui construisent leurs compétences professionnelles dans une alternance terrain-formation (certes très variable selon les académies), accompagnés par des formateurs de l'Institut Universitaire de Formation des Maîtres (IUFM) et des PEMF. Ils sont aussi des professeurs débutants qui construisent leur expérience, et ces débuts dans le métier sont porteurs d'enjeux forts, au plan de leur avenir professionnel comme de la mise en tension de leurs représentations : par leur formation en alternance, ils sont passés d'une approche extérieure de l'école à une approche vécue de l'intérieur, ce qui modifie leurs représentations. C'est cette expérience en construction qui nous intéresse ici en ce qu'elle est propice à l'émergence d'imprévus à prendre en compte.

Notre recherche s'est inscrite dans le cadre de la recherche INRP (2007-2010) menée à l'IUFM de Midi-Pyrénées, école interne de Toulouse 2 Le Mirail, sur l'enseignement de la langue en formation initiale, les convergences et écarts entre activités de formation et pratiques observées dans des classes de stagiaires (Garcia-Debanc et al., 2010).

Les pratiques constatées d'une enseignante débutante en PA, lors d'une séance d'étude de la langue au cycle 3 (grammaire: Proposition Subordonnée Relative), sont analysées dans le cadre psychosocial des imprévus tel qu'élaboré dans nos travaux depuis la thèse en 2001 (e.g. Bénaïoun-Ramirez, 2002, 2009a). Nous nous sommes ainsi intéressés ici aux savoirs disciplinaires et professionnels en jeu, et en particulier aux pratiques langagières de la stagiaire au travers desquelles est visé l'apprentissage de la PSR. En s'attachant plus particulièrement aux imprévus, qui perturbent la personne, auxquels il faut répondre souvent sans avoir le temps de la réflexion, et dont le traitement in situ par l'enseignante débutante donne de la sorte accès à ses représentations de la situation, se pose la question des représentations professionnelles que l'on peut ainsi inférer et de l'identification de traces de modèles de référence qui marquent sa professionnalité ${ }^{1}$.

\section{CADRE CONCEPTUEL : IMPRÉVUS ET « MODĖLES DE PROFESSIONNALITÉ EN ACTES *}

Trois axes structurent notre cadre conceptuel ancré en Sciences de l'éducation et en Psychologie sociale : pratiques et représentations professionnelles, concept d'imprévu.

\section{Les pratiques professionnelles}

Il s'agit de décrire et comprendre les pratiques professionnelles par lesquelles les enseignants visent l'élaboration des savoirs. Les pratiques sont « des systèmes d'action socialement structurés et institués en relation avec des rôles " (Jodelet \& Moscovici, 1990, p. 287). Altet, Bru et Blanchard-Laville (2012) définissent la pratique enseignante comme à la fois les actes observables liés à la pluralité de l'activité professionnelle et les procédés de mise en œuvre d'une activité contextualisée ; ils définissent aussi la pratique professionnelle comme un "faire propre " relationnel, didactique, et des " procédés pour faire » correspondant à une fonction professionnelle, activité telle que définie par le groupe professionnel concerné (p. 13). S'agissant du "faire propre", Bucheton (2009) précise que "l'action découle toujours de décisions prises de manière plus ou moins consciente » (p. 265) et que certains choix renvoient " à la culture et à l'histoire singulière et personnelle des enseignants [... qui] opère comme une sorte de "filtre" pour l'interprétation de la situation (comprendre, apprécier et jouer soi-même la partition) », ces «logiques profondes » fonctionnant en arrière-plan des « représentations possibles de la situation» (p. 46). De même, des auteurs, mettant l'accent sur l'importance du rôle du groupe professionnel pour les pratiques professionnelles, soulignent "l'idée de la pratique comme "façon de faire" " chaque profession possédant ainsi les siennes propres permettant aux professionnels concernés de résoudre les questions liées à l'exercice professionnel (Piaser \& Ratinaud, 2010, p. 10). Les pratiques sont ainsi relatives à un 
objet professionnel, communes à une profession une des conditions de l'existence de représentations professionnelles.

\section{Les représentations professionnelles}

Nous utilisons le concept de représentations professionnelles tel que défini par Bataille (2000; Bataille, Blin, Mias, \& Piaser, 1997), représentations sociales particulières construites dans les contextes professionnels. Portant sur des objets significatifs du milieu professionnel et partagées par les membres de la profession, les représentations professionnelles, dont des éléments peuvent être convoqués selon leur intérêt pour l'action, remplissent diverses fonctions parmi lesquelles celles « d'orientation des conduites » et de "justification anticipée ou rétrospective des pratiques ", cette dernière fonction permettant d'expliquer une action ou de la valider (par exemple dans les discours en situation de formation professionnelle). Bien que s'agissant ici d'un professeur en formation professionnelle initiale, ce concept est pertinent puisque la stagiaire est en exercice sur le terrain.

Pratiques professionnelles et représentations professionnelles sont ainsi en relation dans le champ professionnel. Et si dans la situation d'enseignementapprentissage, les interactions verbales jouent un rôle majeur dans la mise en œuvre effective de l'organisation de l'action (Beguin \& Clot, 2004), nous parlons de «pratiques langagières » en ce qu'elles désignent des pratiques, des manières habituelles de se comporter, utilisant le langage verbal - ou nonverbal - pour témoigner d'une intention de communication en situation d'interaction avec les élèves, afin de transmettre ou faire construire des savoirs. Nous nous intéressons ici aux pratiques langagières, et donc à la fois aux «pratiques verbales » que Trinquier (2009) définit comme des façons de faire ou d'agir se rapportant à des comportements verbaux du maître, des actes de parole récurrents émis par l'enseignant en classe, et au "dialogue pédagogique » (Huber \& Chautard, 2001; Huber, 2007), échange entre l'enseignant et des élèves ou la classe. En situation d'enseignement-apprentissage, les enseignants visent au travers de leurs pratiques langagières l'élaboration des savoirs et utilisent en particulier des reformulations validant ou problématisant les énoncés d'élèves (Volteau \& Garcia-Debanc, 2008).
Pour Grandaty (2005), l'analyse de l'activité langagière montre toutefois que les interventions des enseignants débutants sont souvent destinées à la fois à faire produire à l'élève une réponse précise, attendue, et à distribuer la parole entre les élèves, en cherchant celui qui donnera LA bonne réponse. Il s'établit alors dans les interactions langagières, selon l'auteur, " un statut particulier pour l'élève (être questionné) » grâce auquel l'enseignant demeure «maître du jeu » (p. 51) - voire en posant des « actes de langage » dont le rôle est de «modifier la situation des interlocuteurs » et dont " la force illocutoire » est capitale (Grandaty, 2003, p. 113-114). À travers la nature de telles interactions langagières se construit ainsi « un type particulier de communication où l'enfant ne peut [...] construire son apprentissage sur un oral participatif entre pairs $»$ (2003, p. 114). Et s'il est légitime de penser qu'il s'agit là d'une tentative de réponse aux problèmes professionnels que tout enseignant rencontre, il est tout aussi légitime de s'interroger sur l'ancrage historique de ces pratiques dans des modèles de l'enseignement qui traversent le système éducatif, comme nous le verrons infra.

Des recherches sur les imprévus en classe (Bénaioun-Ramirez, 2002, 2009a) ont mis en évidence que les réponses des enseignants face aux imprévus peuvent relever d' « exigences normatives » auxquelles ils se conforment plus ou moins. Il s'agit de modèles de professionnalité marqués par l'histoire du système éducatif (Hirschhorn, 1993), modèles normatifs de référence profondément ancrés dans la profession, qui surgissent dans «le feu de l'action » et font partie des représentations professionnelles des enseignants : les modèles du « magister " (valeur centrale du savoir) et du "pédagogue» (valeur centrale de l'enseigné) - toutefois nuancés par l'identification d'un modèle de pédagogue "métissé » (Bénaiioun-Ramirez, 2009a). La proposition d'un continuum magister-pédagogue sur lequel l'enseignant se déplacerait en fonction des circonstances, de son histoire professionnelle et de ses implications reste pour nous d'actualité. Bucheton (2009) met ainsi en évidence "le système profond des valeurs qui pilotent l'engagement mais aussi les conceptions de l'apprentissage et l'agir dans la classe » (p. 47). Or relèvent de cet " arrière-plan » les divergences de réponses aux multiples tensions liées par exemple à « l'éthique professionnelle, [...] l'identité professionnelle », l'histoire ou l'engagement (p. 50). Et comme les pratiques ne sont pas totalement transparentes au 
Nicole Bénaïoun-Ramirez

sujet lui-même, il est dès lors licite de penser que certains moments de tensions - et la confrontation aux imprévus en est un, comme nous le montrons infra-peuvent donner accès aux logiques profondes qui pilotent l'agir enseignant en classe. C'est-à-dire, dans notre problématique psychosociale, aux représentations professionnelles que les sujets se sont construites de l'enseignement. Car si la pluralité de l'activité enseignante met en jeu de nombreux facteurs, il est nécessaire, pour faire sens, de réduire la complexité et de choisir un éclairage signifiant pour l'analyse - ici, les modèles de professionnalité.

Ces modèles de professionnalité repérés dans les représentations enseignantes s'ancrent dans des modèles d'enseignement issus de théories de l'apprentissage, décrits par les Sciences de l'éducation et différenciés notamment par la posture de l'enseignant et le statut de l'erreur, ici schématisés :

- modèle de la transmission (Astolfi, 1992/2004), situé sur l'axe du processus «enseigner " (Houssaye, 1993), dans le courant magistro-centriste qui vise la transmission du savoir constitué (Altet, 1998) : posture centrale de l'enseignant, expositive, explicative ; faute à éviter; parfois recherche de LA bonne réponse pour avancer (Grandaty, 2005);

- modèle béhavioriste (Astolfi), situé sur l'axe du processus « apprendre » (Houssaye) : centration sur l'apprenant avec découpage de la tâche et guidage pas à pas « du simple au complexe »; erreur à éviter ;

- modèle constructiviste (Astolfi) - et variantes didactique, socioconstructiviste -, également situé sur l'axe du processus «apprendre » (Houssaye), ou dans le courant centré sur l'apprentissage (Altet) : conception de situations d'apprentissage susceptibles de guider les apprenants dans la structuration du savoir ; erreur constitutive du processus d'apprentissage, à rechercher pour un travail didactique ; parfois un oral participatif entre pairs avec clarification des taches disciplinaire et langagière (Grandaty).

Ces trois modèles d'enseignement constituent une culture commune au groupe professionnel enseignant, dans laquelle chacun puise des éléments plus ou moins à son insu. Ainsi ces modèles, schématiquement décrits, peu étanches dans la réalité des pratiques et complémentaires dans leurs limites de validité (Astolfi, 1992/2004), nous intéressent surtout pour les traces repérables dans les pratiques d'enseignement. Et l'on peut penser que l'imprévisibilité de l'action peut plus particulièrement permettre d'accéder aux représentations professionnelles que les enseignants se sont construites de ces modèles. Ce que nous allons creuser ici à travers ce que nous appellerons "modèles de professionnalité en actes".

Altet, Bru et Blanchard-Laville (2012) évoquent la multidimensionnalité des pratiques enseignantes et en soulignent, parmi d'autres traits constitutifs, la double articulation que travaillent les recherches : à la fois « articulation intention/manières de faire observables » (donc avec prise en compte de la signification pour l'acteur de sa pratique), et « articulation singularité/groupe social » où est en jeu «l'enracinement dans un sujet singulier et dans une tradition (pédagogique, didactique) celle d'un groupe professionnel d'appartenance » (p. 14). C'est bien à cette double articulation que s'attache ce travail sur les « modèles de professionnalité en actes » dont nous pensons que, constitutifs des représentations professionnelles, ils sous-tendent les pratiques, et que l'on peut repérer notamment dans la confrontation aux imprévus. Mais de quels imprévus parlons-nous?

\section{Lintérêt du concept d'imprévu}

Huber et Chautard (2001), identifiant comme imprévus trois types d'incidents (matériels, comportementaux, cognitifs), précisent que l'incident cognitif devient événement lorsque la gestion est plus longue, établissant de la sorte deux niveaux de perturbations, incidents et événements. Pour ces auteurs, l'incident cognitif dans le dialogue pédagogique est un imprévu conçu comme un analyseur de l'activité cognitive des apprenants et comme une variable à piloter et intensifier ; c'est aussi un indicateur de mise en travail des représentations des élèves dans la construction du savoir (Huber, 2007). Bronner (2009) en retient le terme d'incident et le dit « critique » pour définir « l'incident critique » comme imprévu provoquant une perturbation au niveau du projet didactique. Nous ne le suivons pas sur cette voie qui nous semble introduire une confusion avec la définition qu'en donne la psychologie cognitive du travail (Flanagan, 1954), définition reprise en revanche par Loquet, Garnier, et AmadeEscot (2002) qui s'attachent aux apprentissages effectifs (incident critique didactique). 
L'imprévu est aussi défini comme « toute action ou réaction non planifiée par l'enseignant » (Jean, 2008), ce qui survient hors préparation et prévisions de l'enseignant (Jean \& Étienne, 2009). La conceptualisation de l'imprévu par Jean comme triptyque imprévu/ phénomènelévénement - dont le deuxième terme est explicité comme phénomène (2008), ou périphénomène (Jean \& Étienne, 2011) - évoque les imprévus relatifs et radicaux de Perrenoud (1999), les deux niveaux de perturbation de Huber et Chautard (2001) et s'appuie sur les gestes professionnels de Bucheton (2009). L'imprévu y est abordé dans une perspective de formation.

Nous entendons davantage l'imprévu, au sens de l'incident perturbateur de l'Ethnographie de l'École tel que nous l'avons travaillé (Bénaiioun-Ramirez, 2002, 2009a) : ces incidents surgis en classe sans avoir été prévus - et donc dans un contexte d'incertitude pour le sujet - dépendent à la fois du contexte social et du sujet lui-même ; ils perturbent mais l'expérience peut, dans des configurations particulières d'idéal professionnel, d'ancienneté, de contexte et d'implications, jouer un rôle non négligeable quant au caractère « relatif » ou « radical » des imprévus proposé par Perrenoud (1999). Une démarche de co-construction du sens que les enseignants accordent aux imprévus auxquels ils sont confrontés en a permis une formalisation en trois dimensions : contextuelle, pédagogique, didactique. L'imprévu est donc ici abordé du point de vue de l'enseignant, complétant ainsi l'approche de Huber et Chautard. Repéré plus ou moins consciemment par l'enseignant, il se différencie de l'incident critique de Flanagan pointé par l'observateur en fonction d'attentes qui lui sont propres, et cette différence nous permet de travailler la relation que les deux concepts peuvent entretenir avec l'incident cognitif (Bénaïoun-Ramirez \& Panissal, 2010).

Cette approche de l'imprévu a montré que c'est un révélateur (au sens photographique du terme) des représentations que l'enseignant élabore dans cette situation, permettant de comprendre ce qui le perturbe et l'oblige à inventer ses propres réponses, contextualisées, c'est-à-dire dépendant à la fois des ressources et contraintes des contextes de l'enseignement comme de l'apprentissage et des significations qu'il donne à ces contextes (Bru, 1994). Le traitement que l'enseignant met alors en œuvre donne accès à des traces de ses représentations professionnelles qu'il est ainsi possible d'inférer.
Quant aux enseignants novices, attachés aux contenus de leur préparation (e.g. Marcel, 2004), ils sont déstabilisés par les imprévus pédagogiques et n'identifient pas toujours les imprévus didactiques ni l'incident cognitif manifesté dans le dialogue pédagogique (e.g. Bénaioun-Ramirez, 2009a). Nous tenterons dès lors de saisir des moments de mise en tension des représentations. Nous considérons en effet que les représentations professionnelles sont des « processus organisateurs des configurations prises par les pratiques » (Lenoir, 2007, p. 12).

\section{VERS UNE PROBLÉMATIQUE PSYCHOSOCIALE EN SCIENCES DE L'ÉDUCATION}

Ainsi les pratiques langagières des enseignants visant l'élaboration des savoirs les confrontent à des imprévus, en particulier lors des moments de reformulation (Volteau et al., 2008). L'imprévu, contextuel, pédagogique ou didactique, émerge dans un contexte d'incertitude pour l'enseignant, qui signifie alors ce contexte et adapte plus ou moins bien sa conduite (Bénaiioun-Ramirez, 2002, 2009a).

De plus, une fragilité des savoirs peut mettre en difficulté l'enseignant débutant, générant des incidents critiques - repérés par le chercheur (Flanagan, 1954) -, et l'empêcher de prendre en compte des incidents cognitifs (Huber et al., 2001) indiquant la mise en travail des représentations des élèves (Huber, 2007). Ces incidents cognitifs peuvent ainsi constituer des imprévus didactiques (Bénaïoun-Ramirez et al., 2010) dont le traitement par le novice renseigne sur les significations qu'il attribue à la situation et sur ses modèles de référence constitutifs de ses représentations professionnelles, notamment des traces de modèles de professionnalité en actes qui marquent ses pratiques plus ou moins à son insu.

Ce travail ne vise ni à définir ni à prescrire des conduites "efficaces » face aux imprévus. Il tente de décrire et de comprendre les facteurs en jeu qui organisent des pratiques et s'inscrit dans le paradigme de l'interaction contextualisée (Bru, 1994) et notamment l'orientation de la pensée des enseignants développée par Tochon. 
Nicole Bénaïoun-Ramirez

\section{Questions de recherche et propositions}

Quelles représentations professionnelles de l'enseignement peut-on inférer à partir du traitement des imprévus didactiques rencontrés par un enseignant novice pendant la séance? Quelles traces des modèles de référence qui marquent sa professionnalité sont identifiables dans la mise en œuvre de la séance et dans les réponses apportées aux imprévus didactiques? En quoi des modèles de professionnalité en actes pourraient-ils mettre en tension ces représentations ?

Concernant l'étude de cas d'une stagiaire, présentée infra, nous formulons dans une épistémologie de la compréhension ${ }^{2}$, avec «centration sur les processus » (Saada-Robert \& Leutenegger, 2002, p. 21) pour tenter de comprendre des " processus uniques» (e.g. Weisser, 2006, p. 2) à travers une approche qualitative, deux propositions à éprouver :

- P1. Le traitement d'incidents critiques et d'imprévus didactiques par la stagiaire renseigne sur ses représentations de modèles d'enseignement, et plus particulièrement sur ses modèles de professionnalité en actes.

Le repérage des traces de ces modèles rendra attentif à la fois au lien entre incidents (critiques et cognitifs) et imprévus didactiques, et à leur traitement par la stagiaire.

- P2. Lentretien avec le maître-formateur (MF) à l'issue de la séance met au travail les représentations professionnelles de la stagiaire relatives à ses modèles de référence.

Nos données ne nous permettent ni de repérer une évolution des représentations ni de parler de changement des pratiques : un nouveau recueil de données eût été nécessaire quelque temps plus tard. Mais ayant eu accès aux motivations de choix du métier de PES alors qu'elle était encore étudiante, nous pourrons aussi les mettre en relation avec ses discours (y compris lors de l'entretien accordé quelque temps plus tard), et d'éventuelles résistances dans l'analyse de sa mise en œuvre de sa séance - et que ces résistances soient justifiées ou non, tel n'est pas notre propos.

\section{ASPECTS MÉTHODOLOGIQUES}

Les données constituées par une approche clinique concernent une séance d'étude de la langue en cycle 3 (grammaire : la PSR) réalisée en PA par une enseignante débutante (désormais PES). De façon à clarifier la situation, a été passé avec PES et le PEMF (désormais MF) un « contrat» symbolique de communication pour éviter un sentiment de perquisition officielle (De Singly, 1992) : ont été précisés la distinction de postures formatrice-chercheur, l'objet de la recherche et garanti l'anonymat des personnes ainsi qu'un écho de la recherche à MF et un retour à PES, après une auto-confrontation à l'enregistrement vidéo de la séance, le chercheur ne changeant donc de posture qu'en deuxième partie de la rencontre (voir infra).

\section{Des données plurielles}

L'objectif étant de se centrer sur la conduite de la séance de grammaire (PSR) en CM2 par l'enseignante, un enregistrement vidéo a été réalisé avec un caméscope, le plus souvent depuis le fond de la classe, pour éviter de distraire les élèves : 20 mots sont inaudibles, dont plus de la moitié (11) sont toutefois repris le plus souvent par PES ou d'autres élèves.

Cette séance (1h10) retranscrite, avec tours de parole (tdp) numérotés, a été découpée en deux parties et cinq phases :

\begin{tabular}{|c|c|}
\hline \multicolumn{2}{|c|}{ Tableau 1. Verbatim de la séance } \\
\hline \multicolumn{2}{|c|}{$\begin{array}{c}\text { Première partie (42 min } 37) \text { : approche de la notion de PSR } \\
\text { (en trois phases) }\end{array}$} \\
\hline phl (9 min 30) & constitution du corpus : $1-50$ \\
\hline ph2 (20 min 30) & $\begin{array}{l}\text { traitement grammatical du corpus } \\
\text { écrit au tableau : } 51-209\end{array}$ \\
\hline ph3 (12 min 37) & $\begin{array}{l}\text { travail sur la trace écrite (texte à } \\
\text { trous) : } 210-336\end{array}$ \\
\hline \multicolumn{2}{|c|}{ Deuxième partie (27 min 17) : exercices (en deux phases) } \\
\hline ph4 (17 min 17) & $\begin{array}{l}\text { exercices collectifs oraux : } 336-453 \\
\text { ( } 12 \text { min } 57 \text { retranscrites et } 4 \text { min } 20 \\
\text { décrites : exercice collectif conduit } \\
\text { selon les mêmes modalités sans } \\
\text { imprévu notoire malgré la fatigue } \\
\text { des élèves) }\end{array}$ \\
\hline ph5 (10 min) & $\begin{array}{l}\text { exercices écrits : l'enseignante passe } \\
\text { dans les rangs, aucune interaction } \\
\text { langagière avec le groupe-classe. }\end{array}$ \\
\hline
\end{tabular}

Pour respecter l'anonymat des personnes, les prénoms des élèves ont été modifiés (premières lettres et masculin). Ce travail se centrant plus sur la conduite de l'apprentissage que sur l'apprentissage lui-même, il n'a été demandé à PES de communiquer ni les élèves en difficulté ni les résultats des exercices. 
Les documents de préparation de la séance ont été collectés : fiche de préparation de la séance ; trace écrite; deux séries d'exercices dont seule la première, orale et collective, est utilisée ici (des phrases simples aux phrases complexes avec PSR et inversement).

Trois entretiens ont été recueillis :

- un entretien ante (8 min), juste avant la séance, où $\mathrm{PES}$ précise ses intentions didactiques et pédagogiques, comme ses interrogations sur ses choix ;

- un entretien de formation à l'issue de la séance (enregistré et retranscrit, 23 min 54), durant lequel MF demande à PES de donner son ressenti (réussites, améliorations à apporter), lui fait analyser la séance sur des points pour lui essentiels avant de lui faire trouver d'autres démarches possibles ;

- enfin, un entretien d'auto-confrontation réalisé deux mois plus tard, première partie de la rencontre pour visionner l'enregistrement de la séance, non enregistré pour assurer, par un climat plus «banalisé », la spontanéité des réactions de la stagiaire, le chercheur prenant des notes sans commentaires.

Une fiche de présentation, remplie par PES l'année précédente en préparation concours, précise son parcours universitaire, ses motivations de choix du métier de professeur des écoles (perspective humaniste, optimiser les chances de chaque élève de réussir pleinement son projet professionnel et de vie, donner des chances d'ouverture sociale et culturelle) ainsi que ses représentations du métier (valeurs de la République, rôle d'éducation et d'instruction, et d'aide aux élèves à s'adapter). Donnée qui peut permettre une mise en perspective.

\section{Traitement des données}

Une première lecture de l'enregistrement vidéo a permis d'avoir une vue d'ensemble de la séance, la transcription ensuite de l'appréhender plus précisément, enfin plusieurs lectures du verbatim de repérer les incidents critiques et cognitifs ainsi que les divers types d'imprévus auxquels la stagiaire est confrontée et la manière dont elle les traite. Par ce travail d'analyse sont constituées des unités de sens (Bardin, 1991) qui renseignent sur les significations que l'enseignante novice confère à la situation, et ainsi sur ses représentations.

La confrontation aux trois entretiens et aux documents de préparation de la séance permet de mettre en relation les significations inférées de la pratique et des discours pour approcher les représentations professionnelles à travers les traces des modèles de référence en jeu. L'adéquation entre les choix de la stagiaire (objectif de sensibilisation et mise en œuvre), question intéressante au plan didactique, n'est pas traitée ici. Il n'est pas non plus effectué d'analyse didactique a priori de la notion de proposition subordonnée relative. Ce qui nous intéresse ici en effet, ce sont davantage les représentations de l'enseignante novice, sa perception d'éventuelles difficultés face à des imprévus dans la mise en œuvre de sa séance comme le traitement qu'elle en propose. Et cela dans une perspective de compréhension de ce qui se passe pour elle, alors même que les pratiques en contexte sont souvent opaques au sujet lui-même : le recoupement des diverses données nous permet des inférences, dans les limites du cadre conceptuel exposé supra.

\section{DESCRIPTION ET ANALYSE DES RÉSULTATS}

Nous présentons quelques résultats concernant des incidents critiques et imprévus didactiques survenus durant la séance.

\section{Des imprévus et incidents tout au long de la séance}

L'analyse des quatre premières phases de la séance (la $5^{e}$ étant constituée d'exercices écrits) dénombre 6 incidents critiques (IC) et 24 imprévus répartis comme suit :

\begin{tabular}{|l|l|l|l|l|}
\hline \multicolumn{5}{|c|}{ Tableau 2. Iincidents et imprévus dans l'ensemble de la séance } \\
\hline Répartition selon les phases & 6 incidents critiques (IC) & 24 imprévus & 3 pédagogiques & 1 contextuel \\
\cline { 2 - 5 } & & 20 didactiques & 0 & 1 \\
\hline \multicolumn{7}{|c|}{ Première partie : approche de la notion de PSR (ph1 à 3: tdp 1-336) } \\
\hline Phase 1 (ph1), 1-50 & 2 & 2 & 2 & 0 \\
\hline Phase 2 (ph2), 51-209 & 2 & 8 & 0 & 0 \\
\hline Phase 3 (ph3), 210-336 & 2 & 3 & 1 & 0 \\
\hline \multicolumn{7}{|c|}{ Deuxième partie : exercices oraux (ph4: tdp 336-453) } \\
\hline Phase 4 (ph4), 336-453 & 0 & 7 & 1 & \\
\hline
\end{tabular}


C'est en phase 2 (traitement grammatical du corpus) que les imprévus, notamment didactiques, sont les plus nombreux, les IC se répartissant sur les trois phases de la première partie.

Ce nombre élevé d'imprévus en phase 2 s'explique à notre sens par le choix de dispositif exprimé dans l'entretien ante. Les élèves ont en effet à produire (phase 1) des expressions décrivant un portrait («Dame avec éventail», Klimt), pour constituer un corpus de travail, productions que la stagiaire classera au tableau noir sous forme d'un tableau à trois colonnes sans titres. Puis ils confronteront leurs hypothèses d'analyses de ces expressions (phase 2) pour « trouver les titres des colonnes» et indiquer le rôle des Adjectifs Qualificatifs (AQ) et Groupes Nominaux prépositionnels (GNprép) - déjà vu : expansions du nom pour "apporter des précisions» et « enrichir le GN ». Ils approcheront ainsi, par comparaison, la notion de PSR en la resituant dans les expansions du Nom. La démarche est constructiviste (" [se fonder] sur ce que les enfants vont dire»; partir de ce que les élèves savent pour les sensibiliser à la notion de PSR et les aider à construire une compétence de manipulation : repérer, utiliser, transformer), l'entrée choisie fonctionnelle. L'objectif spécifique de la fiche de préparation de la séance est « utiliser» la PSR en « que, qui » « comme expansion $d u$ groupe nominal » mais vu le caractère aléatoire du dispositif la stagiaire envisage d'intervenir dans la production du corpus pour pallier cette difficulté.

Parmi nos nombreuses données, nous choisissons ici de centrer principalement notre attention sur des moments forts de la deuxième phase qui comportent quatre imprévus didactiques et un IC, afin de repérer des traces de modèles de professionnalité en jeu qu'éclaireront d'autres données du recueil.

Une deuxième phase sous le signe de l'imprévisibilité

Un long extrait est ici proposé en plusieurs étapes. Il concerne l'identification des classes grammaticales des AQ et des GNprép et l'approche des PSR, colonnes 1 et 2 (tdp 56-125).
Tableau 3. Extrait de corpus

(Il s'agit d'identifier la classe grammaticale des mots de la colonne 1 - AQ : deux élèves lisent la colonne et un élève chuchote quelque chose)

56 PES : Oui, vas-y, dis-le fort parce que je pense que c'est intéressant.

$57 \mathrm{E}$ Arn : Y a que des adjectifs dans la première colonne.

58 PES : Alors effectivement, c'est bien Arn, tu as devancé ce que je voulais vous faire dire. Il s'agit ici (montre la colonne de gauche), qu'est-ce que j'ai souligné, des.

$59 \mathrm{E}:$ Des adjectifs.

60 PES : Des adjectifs comment.

61 Plusieurs E : Epithètes. (PES dit non, se tourne vers un autre E) Qualificatifs.

62 PES : Des adjectifs qualificatifs. La classe c'est qualificatifs. (écrit en tête de la colonne de gauche, en répétant) Adjectifs qualificatifs.

PES recherche LA bonne réponse qui fera avancer le travail didactique (56) et témoigne en 58 de sa posture constructiviste (faire dire et non dire). Elle veut faire compléter la catégorie grammaticale (60) mais la réponse des élèves (61) est un incident cognitif $(\mathrm{ICog})^{3}$ qui peut témoigner d'une certaine difficulté des élèves sur classe/fonction grammaticales et constitue un imprévu didactique : elle attendait une autre réponse (62), obtenue en 61 par sollicitation d'un autre élève. Ce que l'on retrouve plus loin lorsque PES pose la question de la classe grammaticale des expressions cette fois-ci de la colonne 2 (GNprép) :

88 PES : Alors ici (elle souligne aux cheveux frisés). Arno tu vas d'abord me lire ce qu'il y a marqué s'il te plaît. 89 E Arno : Une dame aux cheveux frisés 90 PES : Oui. [Lecture des autres expressions] 95 PES : Max. 96 E Max : Des adjectifs. 97 PES : Non, c'est pas adjectifs. 98 E : Des compléments circonstanciels. 99 PES : Non c'est pas ça. Vous l'avez vu pourtant. Alors à la place de une dame aux cheveux frisés, je peux dire une dame à les cheveux frisés (écrit à les en tête de la colonne du milieu, encadre le à), enfin c'est pas français d'accord, mais il y a ça dedans (elle montre le à). Qu'est-ce que c'est ça ou bien ça (encadre avec dans la $2^{\mathrm{e}}$ phrase, même colonne). Sim. 100 E Sim : C'est une préposition. 101 PES : C'est une préposition. [...] $102 \mathrm{E}$ Ama : Un groupe nominal. [etc.]

Nous analysons les réponses des élèves en 96 et 98 comme des ICog qui marquent à la fois une réflexion et une incompréhension (Max cherche la classe de frisés et l'élève ce qu'il croit être la fonction de aux cheveux frisés), voire une confusion classe/ fonction (98) en écho à celle de plusieurs élèves 
en 61. Ces ICog constituent un imprévu didactique pour la stagiaire qui convoque alors le souvenir d'un travail antérieur sur les GNprép en l'associant au pointage des prépositions (99) - indice destiné à leur faire trouver qu'il s'agit de préposition $(100,101)$ et de GN (102).

Or plus loin, lors du travail sur le rôle des expressions de la colonne 2 (GNprép), PES introduit un IC qui aura des incidences pour le travail sur le rôle de la PSR (colonne 3) :

Tableau 4. Extrait de corpus

(Travail sur les GNprép de la colonne 2)

105 PES : Alors ça sert à quoi aussi le groupe prépositionnel. C'est quoi sa fonction, vous l'avez vue je crois la fonction du groupe nominal prépositionnel. Oui Tho.

$106 \mathrm{E}$ Tho: C'est quand on dit un dragon avec quelque chose, euh son dragon avec quelque chose.

107 PES : Donc effectivement ça donne peut-être quelques indications, non, parce que un dragon, bon j'ai vu un dragon hier en me promenant, donc il faudrait peut-être le décrire, voyons, alors dis-moi Ama.

108 E Ama : C'est euh.

109 PES: (s'avance vers les dernières tables de la rangée du milieu) Cé, tu penses que ça sert à quoi, les adjectifs ils servaient à quoi (s'arrête devant Cé).

110 E Cé : A dire euh comment est le nom.

111 PES : Oui, et tu crois que le groupe nominal prépositionnel, c'est quoi lui, à quoi il sert, tu sais.

112 E Cé : (silence)

113 PES : (recule d'un rang vers le tableau) Et Lé, tu as une idée.

114 E Lé : (silence).

115 PES : Ah, Tho.

116 E Tho : Ca donne une précision.

117 PES : Ca donne également une précision. Ama.

118 E Ama: On nous a appris que le groupe nominal prépositionnel on pouvait le remplacer par un adjectif.

119 PES : Voilà. Donc tous les deux, d'accord, adjectif qualificatif et groupe nominal prépositionnel servent à enrichir le nom ou le groupe nominal, ce sont des expansions (insiste sur ex-), parce qu'on allonge la proposition (mime l'allongement avec ses mains), ce sont des expansions du nom. Sim.

(Travail sur le rôle des deux expressions de la colonne 3 (PSR), dont la première : la dame qui est dans la forêt).

121 PES : (E Sim lit la 3e colonne) D'accord. Et alors (phrase interrompue).

122 Plusieurs E (coupent la parole à PES, parlent en même temps, certains tout en levant le doigt, brouhaha bref au milieu duquel on entend deux mots) : Complément circonstanciel.

123 PES : Non. Alors j'ai pas posé de questions y a pas de réponse. (silence) Alors ma question est, à votre avis, puisque les adjectifs qualificatifs, puisque les groupes nominaux enrichissent le nom, à quoi sert ce que j'ai écrit dans la troisième colonne. Il va falloir me justifier, aussi. Jof, tu vas y arriver.

124 E Jof : Ça complète le nom.

125 PES : Oui effectivement. (interroge) Qui. Alors, bon j'ai vu, donc je vois une dame, qui est dans la forêt ou dans sa salle de bain c'est pas la même chose. Donc la dame qui est dans sa, la forêt, effectivement, qui est dans la forêt c'est une expansion $d u$ nom, effectivement. Oui. [...]
Alors que les élèves ont déjà fait la confusion classe/ fonction $(61,98)$, PES emploie le mot fonction pour rôle (IC, 105), induisant ainsi une confusion perceptible plus loin en 122. Face à un ICog de Tho (106), PES reformule la réponse (107, indications) et tente de mettre les élèves sur la voie. Mais devant l'incompréhension (108), elle reformule la question (109) et précise ses attentes en donnant comme indice l'allusion au travail sur le rôle des AQ (réponse attendue : expansion complétant le nom). Elle s'appuie sur un nouvel ICog (110) qui est un début de réponse mais, face à un imprévu didactique (silence de Cé, 112), elle donne l'indice d'une similitude avec les GN prép (111) que Tho saisit pour donner un terme voisin de indications proposé plus haut par PES (précision, 116) : réponse acceptée mais insuffisante (117 également). Ama établit un lien (118) avec un apprentissage antérieur, PES en reprend un aspect (119) pour rebondir sur les deux réponses partielles obtenues et donner elle-même la réponse attendue (expansions).

En $121 \mathrm{PES}$ s'apprête à poser la même question du rôle des expressions mais elle est interrompue par un brouhaha de réponses d'élèves qui portent sur la fonction du GNprép (122 Complément circonstanciel) et non sur le rôle de la PSR, ICog que l'IC en 105 a pu induire. PES, surprise par cette confusion entre fonction et rôle (imprévu didactique en 123), répond d'abord au plan pédagogique par un acte de langage, puis au plan didactique en guidant les élèves par des indices (similitude des rôles des AQ et GNprép). En 124 Jof donne une réponse partielle que PES valide comme LA bonne réponse (125 Oui effectivement) mais reformule pour donner elle-même le terme attendu (expansion du nom) tout en l'attribuant à Jof (effectivement. Oui).

Ainsi dans cette conduite du traitement grammatical du corpus produit par les élèves, les pratiques langagières de la stagiaire témoignent d'une fragilité des savoirs disciplinaires, à travers un IC (105) ayant suivi une confusion (ICog classe/fonction en 61-62) ou pu en induire une (ICog rôle/fonction en 122), confusions qui ont constitué des imprévus didactiques. Pour traiter ces imprévus didactiques, et conformément à ses intentions pédagogiques (choix de démarche constructiviste), la stagiaire souhaite faire trouver les éléments de savoirs par les élèves. Mais devant leurs difficultés, et alors même qu'elle souhaite conserver sa posture constructiviste (58), elle leur donne des indices qui transforment la réflexion en jeu de devinette $(107-118,123)$ et 
Nicole Bénaïoun-Ramirez

s'appuie sur LA bonne réponse qu'elle attend (56, 58, $62,101)$, même partielle (117), quitte à la compléter dans une posture expositive $(119,125)$ qui relève du modèle de la transmission, parce qu'elle permet une avancée du temps didactique. D'ailleurs déjà en phase 1 (constitution du corpus) s'est produit un IC lié à la confusion sur la nature de que (pronom relatif ou conjonction de subordination), ce que pointe MF dans l'entretien de formation qui dit craindre chez les élèves une confusion entre relative et complétive sur un exemple comme "je pense que tu es jolie». Fragilité des savoirs professionnels aussi à travers la difficulté à faire construire la notion par les élèves, exprimée plusieurs fois (par exemple 453 « Qui a dit je comprends rien. Ah c'est plus très clair, c'est vrai ») face à l'augmentation des imprévus didactiques en fin de phase 4.

Si ces fragilités sont bien compréhensibles chez un enseignant débutant, elles nous intéressent en ce qu'elles obligent la stagiaire à faire face en situation, en se référant intuitivement à des modèles de professionnalité dont elle s'est construit des représentations, accessibles à travers ses pratiques professionnelles et ses discours.

\section{Des tensions entre modèles de professionnalité}

Ces modalités de traitement des imprévus didactiques relèvent ainsi principalement du modèle traditionnel de la transmission - modèle de professionnalité implicite, inféré de pratiques langagières récurrentes ici en phase 2 (traitement grammatical du corpus). Mais elles révèlent de la sorte un décalage avec un autre modèle de professionnalité auquel elle se réfère explicitement et qui sous-tend ses pratiques, le modèle constructiviste. La volonté de faire découvrir aux apprenants les propriétés du savoir relève bien en effet d'une référence constructiviste de l'apprentissage - et même socioconstructiviste à travers la confrontation souhaitée des points de vue des élèves sur les objets proposés et d'éventuels conflits sociocognitifs, ce qui n'est pas abordé ici. Ce modèle est également sensible dans les pratiques langagières dès la phase 1 de constitution du corpus où, par exemple, sous la pression de la classe PES renonce à faire modifier à l'élève sa proposition :

23 E : La dame qui est dans la forêt. 24 PES : La dame qui est. $25 \mathrm{E}$ : Dans la forêt. 26 PES : Dans la forêt. (va pour écrire au tableau, se retourne vers E) Mais c'est pas une forêt, c'est quoi que tu veux dire. La dame qui. (Plusieurs E parlent en même temps: [inaudible]) 27 PES : D'accord, la dame qui est dans la forêt (écrit la proposition, colonne à droite du tableau, souligne la PSR). D'accord ».

On perçoit ici une manifestation du constructivisme tel qu'elle se le représente, comme ailleurs à plusieurs reprises à travers son insistance à préciser que ce sont les élèves ou le groupe qui ont su trouver la réponse attendue (ainsi 141 On a vu, ou vous avez $v \boldsymbol{u}$, plutôt). Ce sont là des traces de sa représentation du modèle constructiviste, alors même que sa démarche relèverait d'un autre modèle, comme elle le pressent dans l'entretien avec MF à propos des productions des élèves : 36 PES Ben au départ c'était celles des élèves, sauf que je les ai (plus fort, détachant les mots) un-peu-transformées, sans que les enfants ne s'en rendent trop compte. Moment délicat de confrontation des intentions aux pratiques, notamment pour une enseignante débutante en formation.

D'ailleurs, si pendant la phase 4 d'exercices destinée à entraîner les élèves à utiliser le nouveau savoir, un autre modèle, béhavioriste, apparaît souvent, il est implicitement associé à celui de la transmission. Ainsi dans l'échange suivant où un élève doit transformer deux phrases simples en une phrase complexe avec PSR :

398 E Cé : (lit la phrase suivante) Les moineaux du quartier viennent picorer les graines. J'ai déposé les graines dans le nichoir. 399 PES : Donc où est la répétition. 400 E Cé : Les graines. 401 PES : Oui, très bien. Donc ça (montrant au tableau), tu vas le remplacer par un Pronom Relatif, soit qui soit que. (voix montante) D'accord. Alors essaie de voir ce que [inaudible]. Les moineaux du quartier. Vas-y, vas-y. 402 E Cé : Les moineaux du quartier qui. 403 PES : Non vas-y. Viennent. 404 E Cé : Les moineaux du quartier viennent picorer (en même temps que PES) les graines (seule) que j'ai déposées. 405 PES : (en montrant au tableau) Que j'ai déposées dans le nichoir. Très bien, Cé. Très très bien.

Si l'on retrouve dans ce guidage fort « du simple au complexe » visant à instaurer une procédure, et dans sa répétition tout au long des exercices, la marque du modèle béhavioriste, la pratique de l'explication ou information magistrales relève bien de celui de la transmission - sur fond de référence au 
constructivisme (essaie de voir, vas-y quand elle sent qu'elle fait à la place d'élève ; félicite pour l'encourager ou se convaincre qu'il a réussi seul).

L'analyse du traitement des IC et des imprévus didactiques qui témoignent d'une certaine fragilité des savoirs a ainsi permis de mettre en évidence des tensions entre les modèles de référence qui marquent sa professionnalité, constitutifs de ses représentations professionnelles - l'un explicite, "visé ", l'autre implicite, « en actes »- ce qui corrobore notre première proposition. Or l'entretien avec MF questionne ces représentations.

\section{La mise au travail des représentations dans l'entretien de formation}

Pendant l'entretien qui suit la séance, MF pointe à plusieurs reprises la démarche inductrice de PES, comme dans ce passage :

$75 \mathrm{MF}$ : Tu as transformé, ça porte un nom, ça, je veux dire, quand tu. Ils te donnaient une bribe de phrase et toi tu as repris, tu as transformé et tu as fini la phrase. Comment ça s'appelle ça dans la conduite de classe.

76 PES : (chuchotant) C'est transmissif.

77 MF : Oui, c'est transmissif, et puis c'est surtout un mode qui est inductif ${ }^{4}$, on induit, on induit beaucoup, beaucoup, beaucoup.

$[\ldots]$

$81 \mathrm{MF}$ : ... Et donc est-ce que c'est un mode sur lequel on fonctionne comme ça.

$82 \mathrm{PES}$ : Actuellement ce n'est pas préconisé.

$83 \mathrm{MF}$ : Ce n'est pas préconisé et puis est-ce que, c'est pas préconisé bon, il y a les textes officiels, mais pendant qu'on induit, quelle est l'activité réelle des enfants.

84 PES : (rire) Vous induisez ma réponse également.

85 MF : Non, non, je pose une question, quelle est l'activité réelle des enfants.

86 PES : Ma réponse, c'est que donc effectivement ils écoutent et ils ne sont pas acteurs et actifs dans leurs apprentissages.

87 MF : Oui, oui. Mais au-delà des formules, au-delà des formules, qu'est-ce qu'ils font là les enfants, si on se rapporte à la séance.

88 PES : Ben ils écoutent, ils essaient de comprendre. Mais c'est pas eux qui construisent.
$89 \mathrm{MF}$ : Alors est-ce que tous ont bien compris.

90 PES : Non pas tous, c'est compliqué.

L'attitude de la stagiaire (chuchotement en 76, concession en 82 , rire et répartie en 84 , opposition en 86) témoigne d'une certaine résistance. Mais peu à peu elle prend conscience de ses difficultés $(88,90)$ pointées par MF, qui insiste sur l'activité des élèves, et du décalage entre ses ambitions constructivistes et le modèle de professionnalité auquel elle se réfère dans l'action - la transmission $(76,88)$, même associée au béhaviorisme (116 Peut-être au fur et à mesure à force de leur montrer, effectivement c'est tout à fait dans l'inductif...).

Gagnée alors par l'émotion, perceptible à travers le rire (94 (rire) Ben peut-être un peu trop expert) et l'aveu de ses difficultés (116 ...pour le moment je ne sais pas faire autre chose), elle demande de l'aide (164 Mais le problème c'est que c'est une décision qui doit être inconsciente, parce que si vous voulez je le vois) et, face aux conseils de MF (165 Alors il faut essayer... d'ouvrir et de les laisser parler... et toi si tu as ton objectif bien clair derrière la tête, tu sais exactement ce que tu vas pouvoir utiliser... Mais si tu ne leur donnes pas l'occasion, c'est toi qui verrouilles tout), elle résiste encore, invoquant un moyen extérieur (166 Bon mais après il faut que je sache quel exercice peut permettre ça, quelle activité) tandis que MF parle de posture (167 Ah mais c'est pas l'exercice qui le permet, c'est ta posture par rapport aux enfants, qui va induire ça justement ou pas).

On le constate, ses représentations sont ainsi mises en tension, ce que confirme l'entretien d'autoconfrontation quelque temps plus tard. PES reste effectivement d'abord centrée, comme pour se rassurer, sur sa prestance, l'aisance dont elle fait preuve dans sa conduite du groupe. Puis elle prend conscience qu'elle occupe beaucoup l'espace de parole, sur un mode transmissif, mais avoue son inexpérience et sa fragilité quant aux savoirs professionnels en jeu-là : elle ne voit pas comment faire autrement que de donner des explications, comment donner la parole aux élèves pour leur faire construire la notion visée. Il s'agit en effet, pour elle, de tirer parti de la formation pour accorder ses pratiques avec la perspective humaniste qui avait motivé son choix de devenir professeur des écoles.

L'entretien avec MF à l'issue de la séance a effectivement mis au travail ses représentations, ce qui corrobore ainsi notre deuxième proposition. 
Nicole Bénaïoun-Ramirez

\section{DISCUSSION}

Deux principaux modèles de professionnalité, en tant qu'exigences de référence (Hirschhorn, 1993), sont donc en jeu dans les pratiques de l'enseignante novice : un modèle de professionnalité "en actes » implicite (transmission) face aux imprévus didactiques, et un modèle de professionnalité "visé » explicite (constructivisme), perçu comme préconisation de l'Institution, qu'elle viserait pour remplir sa mission de professeur en accord avec son choix humaniste du professorat des écoles qui colore la construction de son appartenance au groupe des enseignants à ce moment de la formation.

Mais le paradigme constructiviste (voire socioconstructiviste) auquel se réfère PES pourrait la questionner sur sa conception des interactions langagières dont nous constatons qu'elle lui permet de s'adresser à un élève chaque fois dans une communication "en étoile». Ce qui, en faisant d'elle le centre des interactions, lui assure la maîtrise du pilotage de l'enseignement quant au savoir en jeu en maintenant l'élève dans un statut de questionné (Grandaty, 2005). Ses interventions semblent en effet destinées à distribuer équitablement la parole tout en cherchant LA réponse attendue pour l'avancée du temps didactique, quitte à poser un acte de langage pour rester maître du jeu. La " coopération langagière entre pairs» (Grandaty, 2003, p. 123) non autorisée, car perçue comme risque de dissipation et de perte d'autorité sur le groupe, et l'opacité des tâches des élèves ne peuvent permettre une " polygestion de l'oral » pour construire des apprentissages, mais les éléments représentationnels du constructivisme mobilisés par la stagiaire présentent l'intérêt de ne pas entrer en conflit avec sa représentation de l'enseignement comme transmission de savoirs : le maître enseigne et les élèves apprennent. Face aux difficultés des élèves dans l'approche de la notion, la novice, qui ne sait comment rebondir sur les incidents cognitifs pour accentuer le travail des représentations des élèves (Huber et al., 2001) mais sait en revanche maîtriser les problèmes de discipline, parvient dès lors à mettre en œuvre sa séance sur un mode principalement transmissif. Identifiant les imprévus didactiques contrairement à d'autres novices rencontrés dans nos travaux, elle ne peut, par fragilité des savoirs, les traiter selon son modèle de professionnalité visé, ce qui interroge la formation.
L'entretien avec MF après la séance pointe le décalage entre son rôle pendant la séance et ses intentions pédagogiques, c'est-à-dire entre modèle de professionnalité en actes et modèle de professionnalité visé. Moment difficile de questionnement de ses représentations, avec apparition de ce qui évoque un "schème étrange » (Flament, 1994) et témoigne de l'importance de la remise en question : ainsi au tdp 138, elle reconnaît sa référence à la transmission (c'était mon tort de vouloir être exhaustive... après peut-être que si j'avais continué des séances avec eux, je me serais rendu compte, je me serais heurtée à leurs difficultés et ça m'aurait fait me remettre en cause et j'aurais essayé de me mettre à leur portée... mais effectivement je ne l'ai pas fait), mais rationalise en invoquant une justification relevant de la transmission (Mais là c'était plus pour poser des outils).

D'un point de vue psychosocial, il s'agit bien là de résistance des représentations au changement. Sans doute parce que le nombre de points à travailler pour un novice peut nourrir la peur de ne pas y arriver (ainsi 163 C'est quelque chose qui est très difficile. Enfin, qui sera difficile pour moi du moins dit PES à MF concernant le rôle des élèves dans le processus d'apprentissage). C'est donc la construction des apprentissages par la coopération langagière entre pairs (Grandaty, 2003), l'institution du groupe en « communauté apprenante » (Maulini, 1999, p. 4), qui apparaît à cette stagiaire à la fois comme une perspective nécessaire en lien avec ses propres finalités et comme une tâche bien lourde. Car « les difficultés de "gestion " ne trouvent leur signification qu'au regard des ambitions pédagogiques du maître » - c'est le sens des deux principales préoccupations des novices (maintien de la discipline et respect du programme). Et «l'expérience aidant, [ils pourront] à la fois mieux dominer ces enjeux et en relativiser l'importance » (Maulini, 1999, p. 4).

Les ambitions pédagogiques de la stagiaire, en cohérence avec le modèle de professionnalité visé, sont ainsi limitées par la confrontation à son principal modèle de professionnalité en actes dans lequel s'ancrent ses représentations professionnelles, convoquées pour leur intérêt dans la confrontation aux imprévus (Bataille et al., 1997). Mais l'entretien avec MF ayant mis au travail ses représentations, on peut penser qu'elle construira avec le temps ses propres réponses aux difficultés qu'elle perçoit, peut-être une complémentarité, dans leurs limites de validité (Astolfi, 1992/2004), entre ses modèles de 
professionnalité, en actes et visé, une compétence de pédagogue "équilibriste » (Houssaye, 1993, p. 21), voire un métissage signifiant pour elle et variable en fonction des circonstances et de ses implications (Bénaiioun-Ramirez, 2009a).

Ce travail sur les modèles de professionnalité en actes proposés ici a cherché à rendre compte non pas de la réalité objective mais d'une réalité vécue, en repérant dans les pratiques d'une enseignante débutante les représentations qu'elle s'est construites des modèles d'enseignement. Ces représentations « organisent " pour partie ses pratiques, les « orientent ", plus ou moins à son insu, et lui permettent, dans cette confrontation à des situations complexes, partiellement imprévisibles, de répondre alors qu'elle ne peut prendre le temps ni de les analyser ni de réfléchir aux conséquences de son action. Comme tout enseignant, elle est ainsi confrontée à la fois à l'incertitude et à la complexité : ses actions échappent à son intention car elles entrent en interaction avec celles des autres acteurs, dans l'incertitude du résultat.

La formation est ainsi interrogée, et particulièrement la formation aux imprévus. Mais sans doute la question se module-t-elle selon la discipline enseignée. Il a été montré ailleurs (Bénaiioun-Ramirez, 2009b) que l'appropriation des savoirs et l'intériorisation de "routines », facilitées par un travail régulier de planification, rendent disponible au travail didactique et autorisent l'improvisation au sens de Tochon. Ainsi pourrait se réduire l'incertitude des situations d'interaction avec les élèves et s'accroître la disponibilité aux imprévus didactiques. Ce qui présente un intérêt pour la formation.

Il serait aussi possible d'élargir ce travail sur la professionnalité par une analyse didactique qui permettrait de croiser modèles de professionnalité en actes et « modèles disciplinaires en acte » (GarciaDebanc, 2007) de manière à observer, par exemple, le rapport de la fragilité des savoirs disciplinaires aux difficultés objectives de la notion et à d'autres modèles de référence à l'œuvre notamment dans les situations de confrontation aux imprévus. Ce qui présenterait également un intérêt pour la formation.

L'observation de séances centrée sur le traitement des imprévus auxquels sont confrontés des enseignants novices est selon nous primordiale pour saisir des moments de mise en tension de représentations professionnelles qui renseignent sur une professionnalité en construction. Complexité qu'une démarche clinique et compréhensive peut aider à approcher. De par les points d'appui qu'elle constitue, la recherche peut ainsi être d'une utilité sociale certaine dans le domaine de la formation des enseignants (Bru, 1994), notamment à un tournant de son histoire.

\section{NOTES}

1. Ce travail se situe ainsi dans le champ de la Psychologie sociale en éducation (et des représentations professionnelles), même si nous évoquons la didactique et convoquons par exemple la notion d'avancée du temps didactique élaborée par les didacticiens.

2. Nous tentons ainsi de comprendre les sujets à partir d'indices, de traces dont nous tentons de mettre à jour le sens (Saada-Robert \& Leutenegger, 2002), comme à partir du sens que prennent leurs actes dans leur univers de référence, et ce sans jugement de valeur.

3. Nous utilisons les abréviations IC pour incident critique et ICog pour incident cognitif.

4. Au sens où les propos de PES, ses questions induisent les réponses attendues des élèves. 
Nicole Bénaïoun-Ramirez

\section{RÉFÉRENCES}

Altet, M. (1998). Les pédagogies de l'apprentissage. Paris : PUF.

Altet, M., Bru, M. \& Blanchard-Laville, C. (dir.) (2012). Observer les pratiques enseignantes. Paris: LHarmattan.

Astolfi, J.-P. (2004). Lécole pour apprendre. L'élève face aux savoirs ( $7^{e}$ éd.). ESF.

Bardin, L. (1991). Lanalyse de contenu. Paris: PUF.

Bataille, M. (2000). Représentation, implicitation, implication : des représentations sociales aux représentations professionnelles. In C. Garnier \& M.-L. Rouquette (dir.), Représentations sociales et éducation (p. 165189). Paris : Éditions Nouvelles.

Bataille, M., Blin, J.-F., Mias, C. \& Piaser, A. (1997). Représentations sociales, représentations professionnelles, système des activités professionnelles. Lannée de la recherche en sciences de l'éducation, 1997, 57-89.

Béguin, P., Clot, Y. (2004). Laction située dans le développement de l'activité, @ctivités, 1 (2), 27-49. Repéré à http://www.activites.org/vln2/beguin.fr.pdf

Bénaiioun-Ramirez, N. (2002). Imprévus et construction de la professionnalité enseignante. Recherche et Formation, 40, 121-139.

Bénaïoun-Ramirez, N. (2009a). Faire avec les imprévus en classe. Représentations professionnelles et construction de la professionnalité. Lyon: Chronique Sociale.

Bénaïoun-Ramirez, N. (2009b). Identités professionnelles entre réflexivité et adaptation : histoires de vie professionnelle de Maîtres-formateurs. Recherche et Formation, 60, 135-150.

Bénaïoun-Ramirez, N., Panissal, N. (2010). Mise en œuvre d'une démarche constructiviste d'enseignement : analyse croisée de corpus. Communication présentée au Congrès international AREF, Genève, Suisse, septembre.

Bronner, A. (2009). Lanalyse du travail didactique du professeur dans la classe. In D. Bucheton (dir.), L'agir enseignant: des gestes professionnels ajustés (p. 159-173). Toulouse : Octarès Éditions.

Bru, M. (1994). Quelles orientations pour les recherches sur la pratique de l'enseignement? L'Année de la recherche en sciences de l'éducation, 1994, 1, 165-174.

Bucheton, D. (2009). (dir.) L'agir enseignant: des gestes professionnels ajustés. Toulouse : Octarès éditions.

De Singly, F. (1992). L'enquête et ses méthodes : le questionnaire. Paris : Nathan.

Flament, C. (1994). Structure, dynamique et transformation des représentations sociales. In J.-C. Abric (dir.), Pratiques sociales et représentations (p. 37-57). Paris : Presses Universitaires de France.

Flanagan, J.C. (1954). La technique de l'incident critique. Revue de psychologie appliquée, avril 1954, 165-185, et juillet 1954, 267-295.

Garcia-Debanc, C. (2007). Les modèles disciplinaires en acte dans les pratiques effectives d'enseignants débutants. In E. Falardeau, C. Fisher, C. Simard, N. Sorin (dir.), La didactique du français, Les voies actuelles de la recherche (p. 43-61). Québec: Presses Universitaires de Laval.

Garcia-Debanc, C., Paolacci, V., Bénaïoun-Ramirez, N., Bessagnet, P., Gangneux, M., Beucher, C. \& Dutrait, C. (2010). Penser la progressivité de l'enseignement grammatical au cycle 3 de l'école primaire : programmations et discours de formateurs et de professeurs des écoles stagiaires. Repères, 41, 201-226.

Grandaty, M. (2003). En quoi un traitement didactique de l'oral transforme-t-il un enfant de petite section de maternelle en élève? Didactiques de l'oral. « Les Actes de la DESco » (p. 112-123). CRDP Basse Normandie, Scérén.

Grandaty, M. (2005). De l'influence des tâches langagières. In L. Talbot (dir.), Pratiques d'enseignement et difficultés d'apprentissage (p. 49-60). Ramonville St-Agne : Édition Erès.

Hirschhorn, M. (1993). L'ère des enseignants. Paris : Presses universitaires de France.

Houssaye, J. (1993). Le triangle pédagogique, ou comment comprendre la situation pédagogique. In J. Houssaye (dir.), La pédagogie : une encyclopédie pour aujourd'hui (p. 13-24). Paris : ESF éditeur.

Huber, M., Chautard, P. (2001). Les savoirs cachés des enseignants. Paris : L'Harmattan.

Huber, M. (2007). Stratégie spontanée et stratégie réfléchie dans la gestion des interactions verbales. In A. Specogna (dir.), Enseigner dans l'interaction (p. 123136). Nancy : Presses Universitaires.

Jean, A. (2008). Peut-on évaluer des compétences au travers des gestes professionnels ? Actes du $20^{e}$ colloque de l'ADMEE-Europe, université de Genève. Repéré à https://plone2.unige.ch/admee08/symposiums/j-s3/js3-4/

Jean, A., Étienne, R. (2009). La gestion des imprévus par un professeur stagiaire. In D. Bucheton (dir.), Lagir enseignant: des gestes professionnels ajustés (p. 93-104). Toulouse : Octarès éditions.

Jean, A., Étienne, R. (2011). L'analyse des traitements des imprévus au service du développement professionnel des enseignants. Communication présentée au Colloque international INRP, mars, Ifé. Repéré à http:// www.inrp.fr/archives/colloques/travail-enseignant/ contrib/36.htm

Jodelet, D., Moscovici, S. (1990). Les représentations sociales dans le champ social. Revue internationale de psychologie sociale, t. 3, 3, 285-288.

Lenoir, Y. (2007). Entrevue avec Marc Bru : Vers une appréhension de la dynamique des pratiques d'enseignement. Formation et profession, 13 (2), 7-13.

Loquet, M., Garnier, A., \& Amade-Escot, C. (2002). Transmission des savoirs en activités physiques, sportives et artistiques dans des institutions différentes : enseignement scolaire, entraînement sportif, transmission chorégraphique. Revue Française de Pédagogie, 141, 99-109.

Marcel, J.-F. (2004). Les pratiques enseignantes de gestion des imprévus. Psychologie et éducation, 56, 31-50.

Maulini, O. (1999). La gestion de classe. Considérations théoriques autour d'une notion bien (trop ?) pratique. 
Repéré à http://www.unige.ch/fapse/SSE/teachers/maulini/classe.html

Perrenoud, P. (1999). Gestion de l'imprévu, analyse de l'action et construction de compétences, Éducation Permanente, 140, 3, Repéré à http://www.unige.ch/fapse/SSE/ teachers/perrenoud/php_main/php_1999/1999_16. html

Piaser, A. \& Ratinaud, P. (2010). Pensée sociale, pensée professionnelle : une approche singulière en Sciences de l'Éducation. Les Dossiers des Sciences de l'Éducation, 23, 7-14

Saada-Robert, M., Leutenegger, F. (2002). Expliquer/ comprendre : enjeux scientifiques pour la recherche en éducation. In F. Leutenegger \& M. Saada-Robert (dir.), Expliquer et comprendre en sciences de l'éducation (p. 7-28). Bruxelles : De Boeck. Repéré à http://www. unige.ch/fapse/publications-ssed/RaisonsEducatives/ REenligne/EXPCOM.html

Trinquier, M.-P. (2009). Approche psychosociale des représentations et des pratiques d'enseignement. Travail et formation en éducation. Repéré à http://tfe.revues.org/ index825.html

Volteau, S., Garcia-Debanc, C. (2008). Les reformulations de l'enseignant dans des interactions orales au cours d'une séance de réécriture de texte littéraire au cycle 3 de l'école primaire. In M.-F. Carnus, C. Garcia-Debanc, A. Terrisse (dir.) : Analyse des pratiques des enseignants débutants : approche didactique (p. 253-272). Grenoble : La Pensée Sauvage Éditions.

Weisser, M. (2006). Expliquer/comprendre : quel paradigme épistémologique pour les Sciences de l'éducation plurielles. Repéré à http://www.inrp.fr/biennale/8biennale/ contrib/longue/75.pdf 